\title{
Kongresse - Tagungen - Events
}

\author{
April 2019
}

\author{
02.04.2019-03.04.2019 \\ BDZV-VERMARKTUNGSGIPFEL \\ BERLIN \\ ZV-Akademie / Premiumpartner: Presse-Versorgung, \\ Branchenlösung Medien
}

04.04.2019 - 05.04.2019

SACM-SGKM-JAHRESTAGUNG 2019

\section{ST. GALLEN/SCHWEIZ}

„Digital Transformation of Media and Communication. Exploring Opportunities and Risk as well as the Stateof-the-art in Digital Transformation of Media and Communication"

Institute for Media and Communication Management at University of St. Gallen

06.04.2019 - 11.04.2019

NAB SHOW - National Association

of Broadcasters (NAB)

LAS VEGAS

$N A B$, die weltgrößte Messe für elektronische Medien

\author{
09.04.2019 \\ LOKALMEDIENTAGUNG \\ ZÜRICH \\ „Neue Chancen für den Lokalmedienmarkt“ \\ Medieninstitut des Verbandes Schweizer Medien
}

\subsubsection{9}

RADIO ADVERTISING SUMMIT 2019

DÜSSELDORF

Audio Trends 2019: „Von UKW bis Smart Speaker“

Veranstalter: Radiozentrale $\mathrm{GmbH}$

\subsubsection{9}

KONGRESS DEUTSCHER LOKALZEITUNGEN 2019

BERLIN

Landesvertretung Rheinland-Pfalz, Berlin

\subsubsection{9}

MEDIENTAGE SPECIAL: CONNECT! THE FUTURE OF TV MÜNCHEN

Medientage München GmbH / Bayerische Landeszentrale für neue Medien / Bayerische Staatskanzlei
Mai 2019

02.05.2019 - 03.05.2019

MEDIENDIALOG HAMBURG 2019

HAMBURG

Freie und Hansestadt Hamburg

08.05.2019 - 10.05.2019

14th GLOBAL BRAND CONFERENCE

BERLIN

„Start-ups, Arts \& Creative Industries:

Fresh Perspectives on Brands"

Journal of Marketing Management (JMM)

06.05.2019-08.05.2019

re:publica 2019

BERLIN

zusammen mit der MEDIA CONVENTION Berlin (MCB)

09.05.2019 - 11.05.2019

64. DGPUK-JAHRESTAGUNG

MÜNSTER

„Integration durch Kommunikation in

digitalisierten Öffentlichkeiten"

Universität Münster (WWU)

12.05.2019 - 14.05.2019

EUROPEAN NEWSPAPER CONGRESS 2019

WIEN

Medienfachverlag Johann Oberauer GmbH

\subsubsection{9}

\section{JAHRESTAGUNG DES ZEK}

RAVENSBURG

„Künstliche und menschliche Intelligenz"

Zentrum für empirische Kommunikationsforschung,

DHBW Ravensburg

\subsubsection{9 - 22.05.2019}

MEDIENTAGE MITTELDEUTSCHLAND 2019

LEIPZIG

"Demokratie, Digitalisierung und Journalismus"

Arbeitsgemeinschaft Medientage Mitteldeutschland e.V.

22.05.2019 - 23.05.2019

KONGRESS DER DEUTSCHEN FACHPRESSE 2019

BERLIN

B2B-Medien-Konferenz 
03.07.2019 - 04.07.2019

HORIZONT DIGITAL MARKETING DAYS 2019

HAMBURG

04.06.2019-06.06.2019

"Der Trend-Check der Digitalbranche"

KÖLN

„Where Broadband meets Content - Fachmesse und Kongress für Breitband, Fernsehen \& Online" ANGA Services GmbH

dfv Conference Group GmbH

August/September 2019

05.06.2019-07.06.2019

EMMA 2019 CONFERENCE

LIMASSOL/ZYPERN

European Media Management Association

„Media Management and Actionable Knowledge:

The Relationship between Theory and Practice"

24.06.2019

MEDIA TASTING

STUTTGART

Tastings, Podium, Fishbowl, Face2Face

26.06.2019 - 27.06.2019

SCREENFORCE DAYS 2019

KÖLN

"Größter Branchenevent für Bewegtbild“

Sceenforce Gattungsmarketing GmbH

Juli 2019

02.07.2019-03.07.2019

LOKALRUNDFUNKTAGE 2019

NÜRNBERG

Deutschlandweit größter Branchentreff für den

lokalen und regionalen Rundfunk

Medientage München GmbH / Bayerische

Landeszentrale für neue Medien /

Bayerische Staatskanzlei

03.07.2019-05.07.2019

SÜDDEUTSCHER DRUCK- UND MEDIENTAG 2019

ULM

Verband Druck und Medien Bayern

20.08.2019-24.08.2019

GAMESCOM

KÖLN

Koelnmesse GmbH

06.09.2019 - 11.09.2019

INTERNATIONALE FUNKAUSSTELLUNG

BERLIN

Messe Berlin GmbH 